\title{
Analysing the Kinematic Characteristics of Bennett Mechanisms and Its Networks for Usage in Forming Deployable Structures
}

\author{
Tony Punnoose VALAYIL*, Selladurai VELAPPAN**, Prakash Lakshmana PANDIAN*** \\ *Coimbatore Institute of Technology, Coimbatore-641014, India, E-mail: tonyisdesigning@gmail.com \\ **Coimbatore Institute of Technology, Coimbatore 641014, India, E-mail: selladurai.v@gmail.com \\ ***Coimbatore Institute of Technology, Coimbatore-641014, India, E-mail: clpprakash@gmail.com

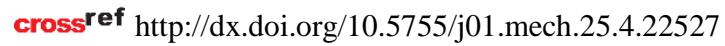

\section{Introduction}

Bennett mechanism was invented by G.T. Bennett in 1903 [1]. "Its direct application in the ordinary business of practical machine design has been ...nil, but its continuing importance for our understanding of the over-constrained yet mobile linkages is immense," says Dr. Jack Phillips in his treatise on machinery (sec. 20.53), after discussing about the Bennett linkage in the treatise [2,3].

Bennett mechanism is a spatial four-bar mechanism having four kinematic links and four revolute joints. It has a closed-loop kinematic structure. All the links are twisted, which means twist angle is present in all the links of the Bennett mechanism, thus making it as a spatial mechanism. The degree-of-freedom of this mechanism is one. It finds applications in deployable tents, long span shelters and in structures where saddle surfaces are required.

Researchers and scientists were engrossed on Bennett mechanism and its special characteristics, especially its kinematic characteristics. Chen and You [4] proved mathematically the existence of special forms of Bennett linkages and its applications. Lee [5] derived the angular displacement, angular velocity, angular acceleration and coupler curve equations for the Bennett mechanism. Lee also performed the dimensional synthesis of Bennett mechanism. Lee also discussed about cross Bennett and open Bennett and the various conditions for its existence. The influence of twist angle on the displacement factor, velocity and acceleration factors was done by Zhi et al. [6], also the influence of twist angle on the driving moment, reaction forces and moments of the joints were also analyzed. A scissor-like Bennett mechanism was designed by Wei [7] and also developed the conditions for the existence of equilateral Bennett mechanism. Parkin [8] used a finite displacement screw method to explain Bennett linkage characteristics and also found the singularity configuration of Bennett mechanism. Brown and Milenkovic [9] replaced one of the revolute joints of Bennett mechanism by a spherical joint and obtained a lower-over-constraint form of the Bennett linkage. Baker and Min [10] tried connecting two Bennett linkages together, but obtained a rigid one. Baker [11] related all four joints and four links of the Bennett mechanism with J-Hyperboloid and L-Hyperboloid. Yang [12] developed and analysed the symmetrical properties of a deployable Bennett network having only one degree-of-freedom. The network obtained had a saddle surface and not an accurate hyperboloid nor an accurate hyperbolic paraboloid surface. Baker [13] examined the reasons for a collapsible skew network composed of Bennett linkages. Bil [14] proposed that the Bennett mechanism consist of two dyads.
By considering the various reviews presented above, the idea of two types of Bennett mechanisms and making networks out of these two types came into this research.

In this paper, section 2 focuses on the first type of Bennett mechanism and its characteristics. This has two kinematic links with equal lengths. Then section 3 focuses on type 2 Bennett mechanism or equilateral Bennett mechanism and its characteristics. Section 4 focus on coupler curves of both the types of Bennett mechanism. Section 5 focuses on kinematic analysis-angular displacement, angular velocity and angular acceleration of Bennett mechanism and section 7 focus on making networks using the two types of Bennett mechanisms. Section 8 provides the results of all the sections and section 9 concludes the work.

\section{Characteristics of type 1 Bennett mechanism}

This type of Bennett mechanism has four links, in which, the opposite links are of equal length. The schematic diagram of type 1 Bennett mechanism is shown in Fig. 1.

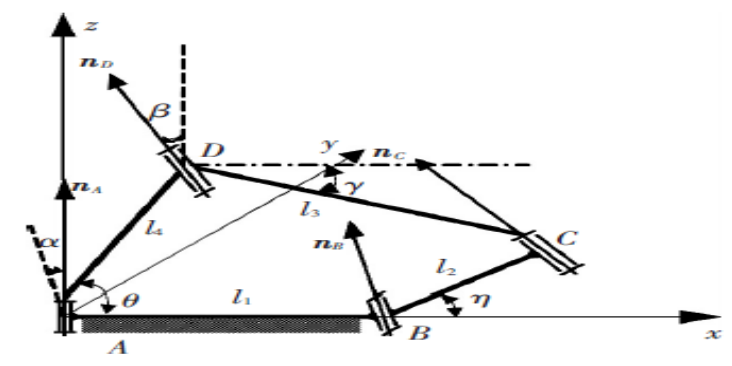

Fig. 1 Bennett mechanism [6]

The Bennett mechanism ABCD shown in Fig. 1 has non-parallel and non-intersecting four revolute joint axes [6]. The two opposite links have the same length and same twist angles [6]. Therefore:

$$
\begin{aligned}
& D C=l_{3}=A B=l_{1}, A D=l_{4}=B C=l_{2}, \\
& D C=A B=\alpha \text { and } A D=B C=\beta,
\end{aligned}
$$

where: $l_{1}, l_{2}, l_{3}, l_{4}$ represent lengths of the four links of the type 1 Bennett mechanism. $\alpha$ and $\beta$ represents twist angles in the opposite links of the mechanism.

Another condition for the existence of type 1 Bennett mechanism is that the opposite link lengths and twist angles should satisfy the equation [6]

$$
\frac{\sin \alpha}{l_{1}}=\frac{\sin \beta}{l_{2}} \text {. }
$$


Using the above-mentioned conditions, a physical model of type 1 Bennett mechanism was constructed using Polyvinyl chloride pipe (PVC). Then two of its kinematic links are connected using a nut and bolt to make it a turning pair. The turning pair will have one degree-of-freedom and this arrangement is shown in Fig. 2, a. The z-axis points along the axis of rotation for revolute joints. Then using the same above-mentioned conditions, a 3D model of the type 1 Bennett mechanism was developed in MATLAB as well and is shown in Fig. 2, b.

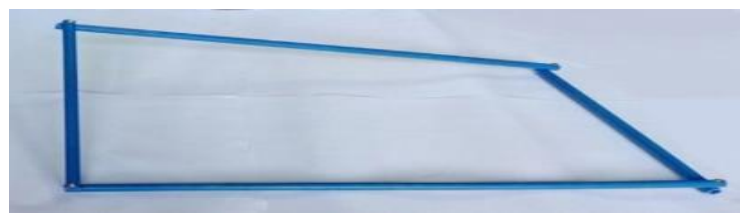

a

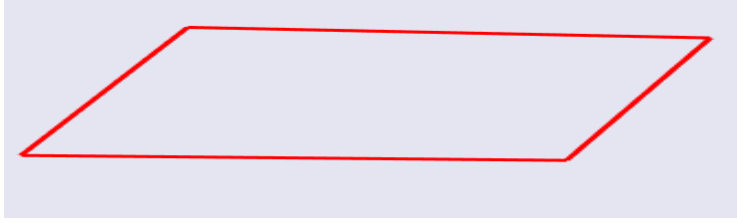

b

Fig. 2 Type 1 Bennett mechanism (a) physical model, (b) MATLAB model

\section{Characteristics of type 2 Bennett mechanism}

Type 2 Bennett mechanism is also known as equilateral Bennett mechanism. A physical model which was constructed using PVC and the MATLAB model of equilateral Bennett mechanism is shown in Fig. 3, a and b.

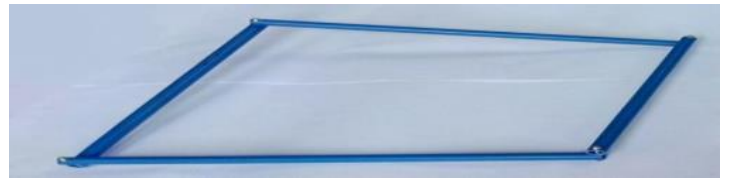

a

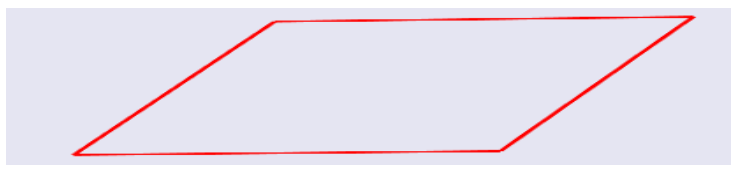

b

Fig. 3 Equilateral Bennett mechanism (a) physical model (b) MATLAB model

The conditions for the existence of equilateral Bennett mechanism is given by [12]:

$$
\begin{aligned}
& \alpha+\beta=\pi \\
& l_{1}=l_{2}=l_{3}=l_{4},
\end{aligned}
$$

where: $l_{1}, l_{2}, l_{3}, l_{4}$ represent lengths of the four links of the equilateral Bennett mechanism. $\alpha$ and $\beta$ represent twist angles present in the opposite links of the equilateral Bennett mechanism.

\section{Coupler curves for type 1 and type 2 Bennett mecha- nisms}

Coupler curve is a path traced by any point on the coupler link. Coupler link is a link which connects the input link and the output link. In Fig. 1 DC is the coupler link. It is very important to find the path traced by a point on the coupler link because in many simple mechanisms having only four links, this path denotes the output for the application to be used. Information about coupler curve is also important for solving path generation and rigid-body guidance problem [15]. In order to plot the coupler curve for the type 1 and type 2 Bennett mechanisms, the coordinates for all the four revolute joints are found out. Now consider the type 1 Bennett mechanism ABCD (as in Fig. 1) for coupler curve analysis. Let's attach a fixed reference frame at $\mathrm{A}$. Then the joint coordinates are obtained as [6]:

$$
\begin{aligned}
& A(0,0,0), B\left(l_{1}, 0,0\right), D\left(l_{2} \cos \theta, l_{2} \sin \theta, 0\right) \\
& C\left(\left(l_{1}+l_{2} \cos \eta\right),\left(l_{2} \cos \alpha \sin \eta\right),\left(l_{2} \sin \alpha \sin \eta\right)\right)
\end{aligned} .
$$

The path traced by point $\mathrm{C}$ can be considered for plotting the coupler curve for the above-mentioned Bennett mechanism. Therefore the input angle $\theta$ and the output angle $\eta$ are calculated by using the equation [6]:

$$
\eta=\left\{\begin{array}{l}
2 \pi+2 \arctan \left(K \tan \frac{\theta}{2}\right), \theta \in[0, \pi] \\
2 \arctan \left(K \tan \frac{\theta}{2}\right), \theta \in[\pi, 2 \pi]
\end{array}\right\},
$$

where:

$$
K=-\frac{\sin (\beta \pm \alpha)}{\sin (\alpha)-\sin (\beta)} .
$$

The coupler curve is plotted using MATLAB and is shown in Fig. 4.

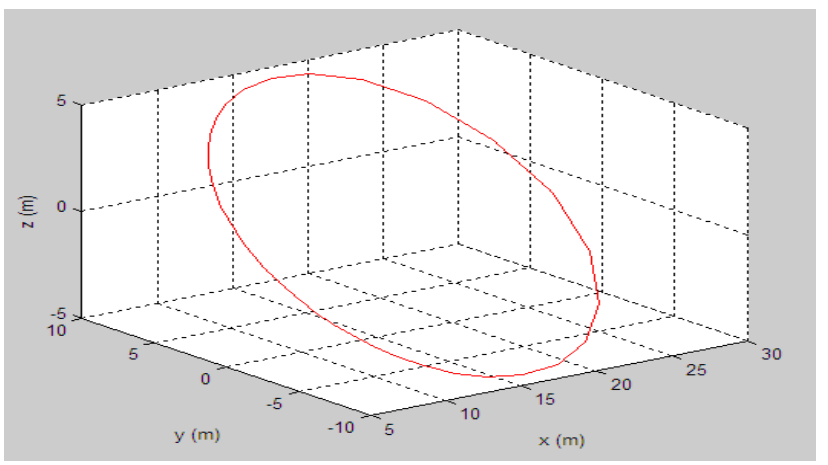

Fig. 4 Coupler Curve for type 1 Bennett mechanism

The above coupler curve indicates that the point $\mathrm{C}$ moves in a three-dimensional space for each and every variation of the input angle $\theta$ from $0^{0}$ to $360^{\circ}$.

Similarly, the coupler curve for equilateral Bennett mechanism can be plotted easily by substituting $l_{1}=l_{2}$ and $\alpha+\beta=\pi$ (these equations forms the conditions for its existence as mentioned in section 3 ) in the above equations. 
Path traced by point $\mathrm{C}$ of type 2 Bennett mechanism is shown in Fig. 5.

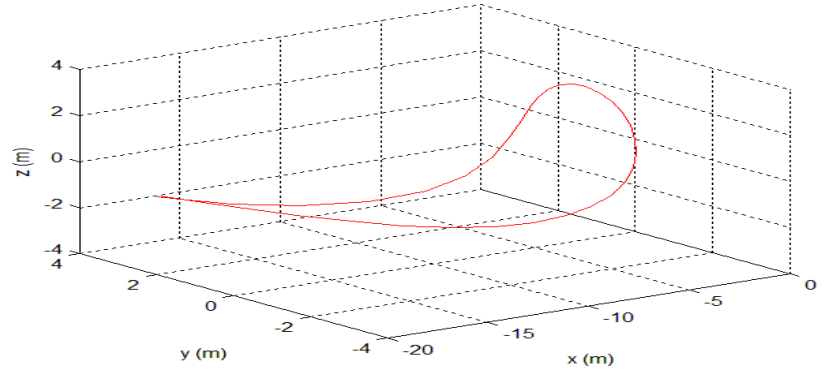

Fig. 5 Coupler curve for equilateral Bennett mechanism

The results presented in Figs. 4 and 5 indicates that the point $\mathrm{C}$ moves in a three-dimensional space making both type 1 and type 2 Bennett mechanism a spatial mechanism. Also, it is clear from both the figures that type 1 and type 2 Bennett mechanisms have different output motions and hence for solving the path generation problem different strategies have to be followed for both the types of Bennett mechanism.

\section{Kinematic analysis - angular displacement, angular velocity and angular acceleration of Bennett mecha- nisms}

Input-output displacement equation can be obtained from the length of the coupler link CD by considering the coordinates of the joints $\mathrm{C}$ and $\mathrm{D}[6]$.

$$
\begin{aligned}
& \cos (\theta) \cos (\eta)+\left(\frac{l_{1}}{l_{2}}\right)(\cos (\theta)-\cos (\eta))+ \\
& +\cos (\alpha) \sin (\theta) \sin (\eta)=1 .
\end{aligned}
$$

A graph is plotted between the input angle $\theta$ versus the output angle $\eta$ in MATLAB and as shown in Fig. 6.

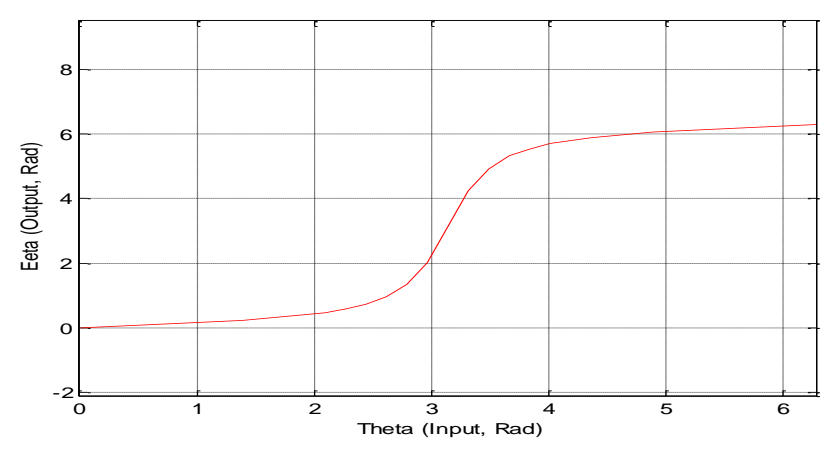

Fig. 6 Angular displacement graph ( $\theta$ vs. $\eta$ ) anism.

The graph (Fig. 6) is for the type 1 Bennett mech-

The application of the half-angle relations to above Eq. (5) relates to the following equation [6]:

$$
f_{1}(\theta) x^{2}+2 f_{2}(\theta) x+f_{3}(\theta)=0
$$

where:

$$
x=\tan \left(\frac{\eta}{2}\right)
$$

$$
\begin{aligned}
& f_{1}(\theta)=\left(\left(\frac{l_{1}}{l_{2}}\right)-1\right)(\cos \theta+1) \\
& f_{2}(\theta)=\cos \alpha \sin \theta \\
& f_{3}(\theta)=\left(\left(\frac{l_{1}}{l_{2}}\right)+1\right)(\cos \theta-1)
\end{aligned}
$$

By using the above equations, it yields two solutions for $\eta$ as follows [5]:

$$
\eta=2 \tan ^{-1}\left\{\frac{-f_{2}(\theta) \pm \sqrt{f_{2}(\theta)^{2}-f_{1}(\theta) f_{3}(\theta)}}{f_{1}(\theta)}\right\} .
$$

The \pm sign in the above equation can be interpreted as representing two kinds of Bennett mechanisms as shown in Fig. 7.
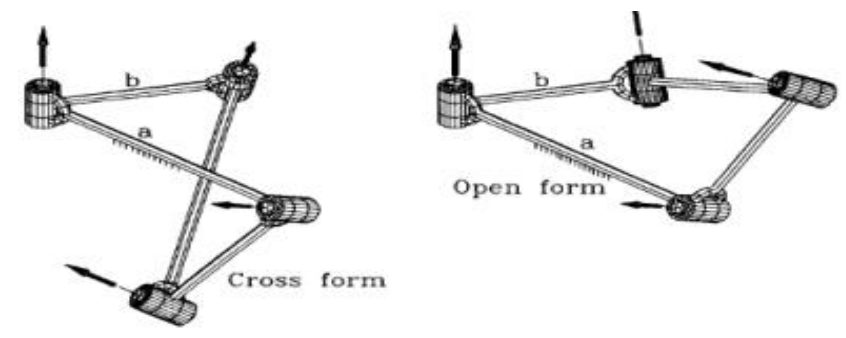

Fig. 7 Two kinds of Bennett 4R mechanisms [5]

The plus indicates an open type and the minus indicates the cross type. The above equation for $\eta$ can be rewritten as [5]:

$$
\frac{\tan \frac{\eta}{2}}{\tan \frac{\theta}{2}}=\frac{\cos [(\alpha-\beta) / 2]}{\cos [(\alpha+\beta) / 2]}
$$

or

$$
\frac{\tan \frac{\eta}{2}}{\tan \frac{\theta}{2}}=-\frac{\sin [(\alpha+\beta) / 2]}{\sin [(\alpha-\beta) / 2]}
$$

where: $\frac{l_{1}}{l_{2}}= \pm \frac{\sin \alpha}{\sin \beta}$ and

$$
\tan \frac{\theta}{2}=\frac{\sin \theta}{(1+\cos \theta)} .
$$

In which angles $\alpha$ and $\beta$ may be acute or obtuse, and positive or negative.

Differentiating the displacement equation (5) with respect to time the angular velocity ratio can be obtained as [5]:

$$
V r=\frac{\dot{\eta}}{\dot{\theta}}
$$

where: 


$$
V_{r}=\left(\frac{\frac{l_{1}}{l_{2}} \sin \theta+\sin \theta \cos \eta-\cos \alpha \cos \theta \sin \eta}{\frac{l_{1}}{l_{2}} \sin \eta-\cos \theta \sin \eta+\cos \alpha \sin \theta \cos \eta}\right) .
$$

This equation is known as angular velocity equation. A graph is plotted between the input angles $\theta$ vs. the angular velocity $V_{r}$. The obtained graph in MATLAB is as shown in Fig. 8.

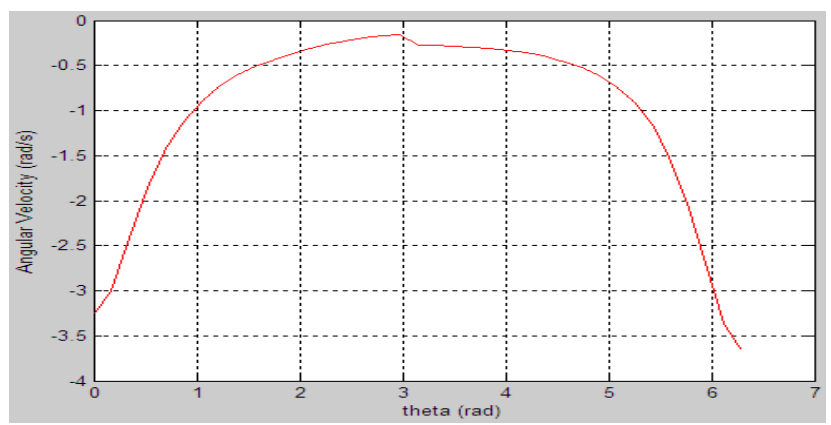

Fig. 8 Relation between Angular Velocity vs. $\theta$

Angular acceleration can be obtained if we again differentiate the displacement equation (5) with respect to time [5]:

$$
\begin{aligned}
& \ddot{\eta}=D_{1}(\theta) \times \\
& \times\left\{\dot{\theta}^{2}\left[D_{2}(\theta) V_{r}^{2}-2 D_{3}(\theta) V_{r}+D_{4}(\theta)\right]+\ddot{\theta} D_{5}(\theta)\right\},
\end{aligned}
$$

where:

$$
\begin{aligned}
& D_{1}(\theta)=\left(\frac{1}{\left(\frac{l_{1}}{l_{2}} \sin \eta-\cos \theta \sin \eta+\cos \alpha \sin \theta \cos \eta\right)}\right), \\
& D_{2}(\theta)=\left(1-\frac{l_{1} \cos \theta}{l_{2}}\right), \\
& D_{3}(\theta)=(\sin \theta \sin \eta+\cos \alpha \cos \theta \cos \eta), \\
& D_{4}(\theta)=\left(1+\frac{l_{1} \cos \eta}{l_{2}}\right), \\
& D_{5}(\theta)=\left(\frac{l_{1}}{l_{2}} \sin \theta+\sin \theta \cos \eta-\cos \alpha \cos \theta \sin \eta\right) .
\end{aligned}
$$

Then a graph is plotted between the input angles $\theta$ vs. the angular acceleration $\dot{\eta}$ ). The obtained graph from MATLAB is as shown in Fig. 9.

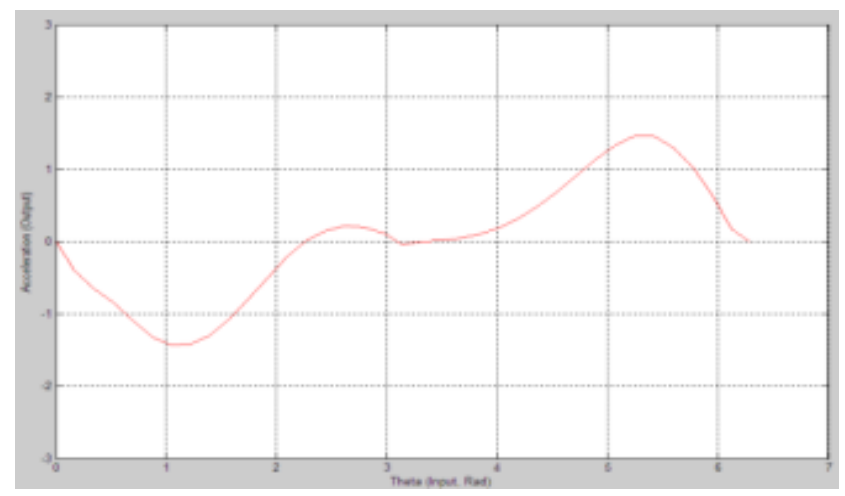

Fig. 9 Relation between Angular Acceleration vs. $\theta$

\section{Bennett networks}

A group of Bennett mechanisms are interconnected to form a network. The way of interconnecting plays a major role in forming a network. Here a single Bennett mechanism is taken and its entire links are extended. Then the remaining Bennett mechanisms are connected to these extended links. Therefore on actuating the two adjacent extended links the entire network gets actuated. Instead of using extended links, if we use normal links then each row of the network should be actuated separately.

In this paper, the network is made only by using extended links and hence a single actuation is alone needed to operate.

A network of Bennett mechanism was designed to analyze its mechanical behavior. To achieve this, a Bennett linkage is constructed based on the geometrical conditions and the kinematics of the mechanism. A Bennett network with 9 identical Bennett mechanisms were built physically by using Polyvinyl chloride (PVC) pipes and joints with nut and bolt which acts as a one degree-of-freedom joint. The position of each joints were calculated mathematically and then the network was simulated in MATLAB.

As shown in Fig. 10, the mechanisms are arranged in such a way that the one Bennett mechanism having extended links was placed at the centre and the remaining 8 mechanisms were connected surrounding it, so that on actuating the extended links the entire network gets actuated. This is illustrated in Fig. 10.

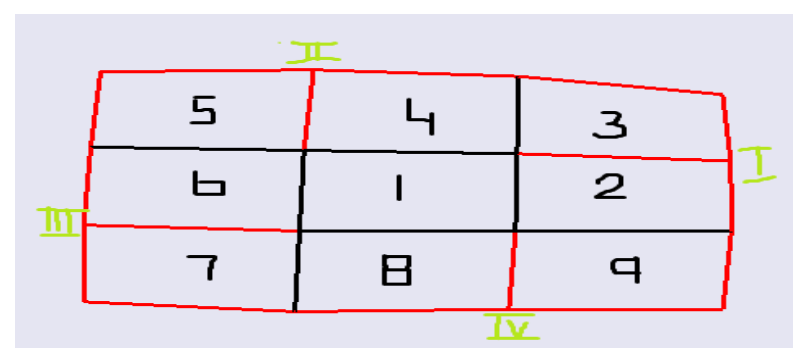

Fig. 10 A Bennett network developed in MATLAB

In order to construct a network using Bennett linkages, one Bennett linkage with extended links marked with number ' 1 ' and shown in black lines is put in the centre which satisfies all the Bennett conditions are shown in Fig. 10. All other Bennett linkages with identical geometric parameters like linkage 1 are arranged surrounding linkage 1. It means that all vertical links and all horizontal links are in the same parameters respectively. In this network, each thick black line represents a rigid link extended from linkage 
1. The segment of each thin red line also represents a rigid link. The intersection points of thick or thin lines in network are all revolute joints for Bennett linkages. In this way, the network of Bennett Linkage is formed.

Type 1 and type 2 Bennett mechanisms are used to construct networks. Therefore two Bennett networks are obtained. To solve the problem let us consider first the type 1 Bennett mechanism.

To construct a network using type 1 Bennett mechanism, the link lengths are taken as $l_{1}=167.7 \mathrm{~cm}$ and $l_{2}=100 \mathrm{~cm}$. Twist angles are taken as $\alpha=30^{\circ}$ and $\beta=17.46^{\circ}$. By using this data, a physical model of a Bennett network using type 1 Bennett mechanism is constructed and shown in Fig. 11.

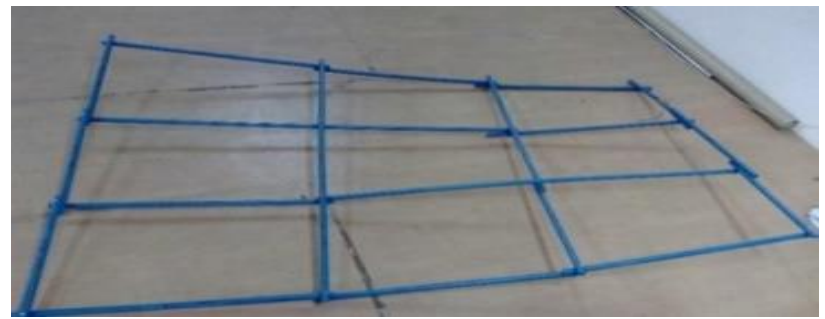

Fig. 11 Constructed model of a Bennett Network using type1 Bennett mechanism

To determine the mobility of the Bennett network, inner angles of linkage 1 can be taken as the input angle. As Bennett linkage has mobility one, linkages 2, 4, 6, and 8 in the network are driven by linkage 1 through their extended links. The four extended links of the linkage 1 divide the whole network into four regions, I, II, III, and IV. Therefore in the region I, linkages 3 is driven by the extended links of linkage 1 and the links of linkage 2 and so on for other linkages of other regions. Through such kinematic transform, the whole network can be driven uniquely by the input in linkage 1 . Therefore, all the Bennett linkages in the network are compatible to each other. And the network has a mobility of one.

The coordinates of all the joints in the network are calculated using type 1 bennet mechanism for three different input angles of $\theta\left(\theta=30^{\circ}, \theta=60^{\circ}, \theta=90^{\circ}\right)$. The network was drawn for these configurations in MATLAB and different shapes of the network are obtained in Fig. 12.

Comparison of type 1 physical model with the type 1 MATLAB model can done using Fig. 11 and Fig. 12, c. In Fig. 11, saddle surface formation can be observed clearly.

In the second model i.e. replacing opposite equal links with all equal links. The configuration parameters are $l_{1}=l_{2}=100 \mathrm{~cm}$ and $\alpha=30^{\circ}$. The coordinates of all the joints are calculated for two different input angles of $\theta\left(\theta=30^{\circ}\right.$ and $\theta=60^{\circ}$ ). The network was drawn for these configurations in MATLAB and different shapes of the network are obtained and are shown below in Fig. 13, a and b. For $\theta=90^{\circ}$ we get the same configuration as shown in Fig. 12, c.

\section{Different cases}

By changing the twist angle, the shape of the network is analysed:
Case 1: With $\alpha=20^{\circ} l_{1}=167.7 \mathrm{~cm}, l_{2}=100 \mathrm{~cm}$.

Case 2: With $\alpha=30^{\circ} l_{1}=167.7 \mathrm{~cm}, l_{2}=100 \mathrm{~cm}$.
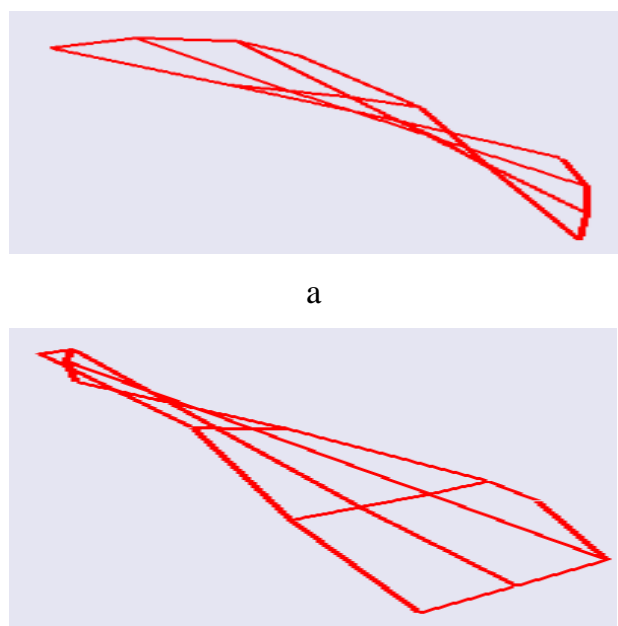

b

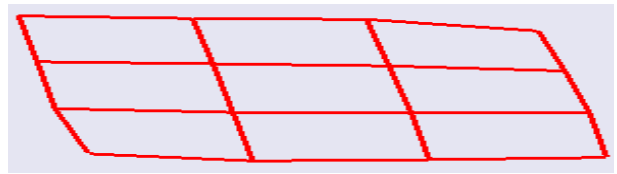

Fig. 12 Type 1 Bennett Network. (a) Network for $\theta=30^{\circ}$. (b) Network for $\theta=60^{\circ}$. (c) Network for $\theta=90^{\circ}$
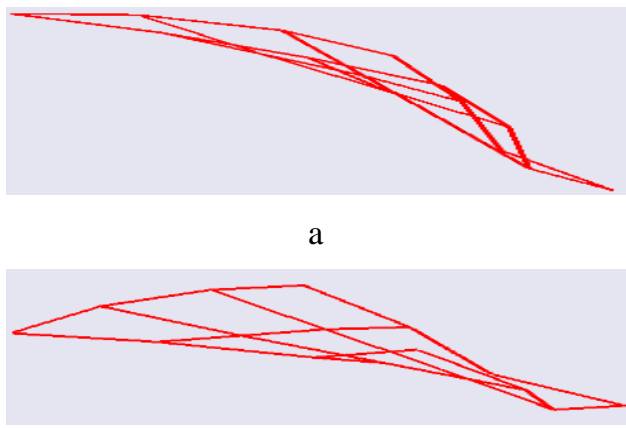

b

Fig. 13 Type 2 Bennett Network. (a) Network for $\theta=30^{\circ}$. (b) Network for $\theta=60^{\circ}$

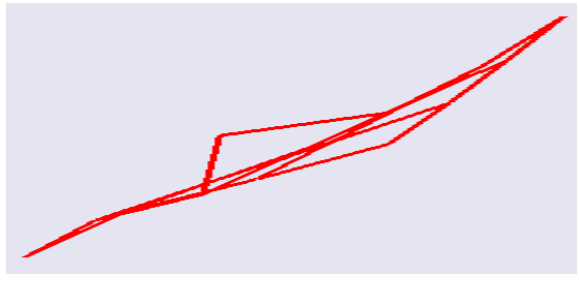

a

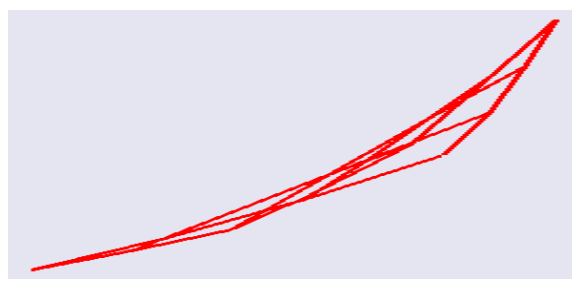

b

Fig. 14 Saddle Shape formation (a) Case 1 (b) Case 2 
By analysing the shape of the networks in the above Figs. 14, a and b, it can be observed that the shape of the network for different twist angle, but same input angle, it gives a saddle surface. By increasing the twist angles, the saddle structure is formed perfectly. Thus twist angles are the main reason for forming these saddle structures using Bennett mechanism.

A saddle shape has opposite curvatures, a downward arch in one direction and an upward arch in another direction. Obtaining the perfect saddle is very difficult when constructing at a plane higher than the lower ground level. Hence to obtain a perfect and expected saddle shape, the required saddle structure is designed by choosing the appropriate link length and twist angle. If there is any change in the design, the design can be altered and then the design can be constructed at the lower level. Note that only the base frame is alone constructed. Since it is a deployable structure, it can be folded and then raised to the top floor and then expanded and then the movements of the links are arrested so that it does not fold. This forms as a base for the saddle structure and hence it makes the work easier in building the saddle roofs.

\section{Results}

Two types of the Bennett mechanims are constructed, both physically and in MATLAB i.e. one with opposite links of equal length and another with all the links of equal length. Former is called as the type 1 Bennett mechanism and the later is called as type 2 Bennett mechanism. From section 2 and 3 presented in this paper, it is found that the working range for both the models is equal to $145^{\circ}$, but the minimum folding angle differs. For type 2 Bennett mechanism, the minimum folding angle is $7^{\circ}$, but for type 1 it is $9^{\circ}$. Hence the foldability of using type 2 Bennett linkages is high and hence it is more compact than type 1 Bennett mechanism. Type 2 Bennett mechanism is also known as equilateral Bennett mechanism.

From Section 5 presented in this paper it can be concluded that, in the 2 types of Bennett mechanism, the influence of twist angle $\alpha$ on displacement factor is very less or negligible. It is to be noted from Fig. 6 and Fig. 9 that the plot is asymmetrical at $\theta=\eta=\pi$ Thus the results show that twist angle $\alpha$ has few influence on the displacement factor and has a greater influence on the velocity and acceleration factors.

Then Bennett networks were constructed both physically and in MATLAB using both the types of Bennett in section 6. On analysing the two kinds of developed Bennett networks, the shape of the structure was found to be similar to that of a saddle. It was found that the network is a deployable structure and has a shape of the saddle structure. The steepness of the saddle shaped structure formation was analysed by varying the twist angles and keeping the link length as constant. It is found that the steepness of the saddle surface formation is obtained with the increase in twist angle

From the two configurations of Bennett network, it is found that the input angle working ranges differ for both type 1 and type 2 Bennett mechanism. Input angle working range for type 1 is from $30^{\circ}$ to $149^{\circ}$ and the input angle working range for type 2 is from $21^{\circ}$ to $140^{\circ}$. This shows that working range for both the networks is $119^{\circ}$ and therefore working with type 2 is more compatible than working with type 1. After analysing the shape of the network, it is found that the shape of the network for any input angle creates a saddle like surface.

Thus this network operates similar to that of a deployable structure i.e. the network becomes more compact when the angle between the adjacent extended links (black colour links seen in Fig. 10) are less, the network gets expanded when the angle between the extended links increases up to $90^{\circ}$, after $90^{\circ}$ the network starts shrinking. From Fig. $12 \mathrm{a}, \mathrm{b}$ and $\mathrm{c}$, as $\theta$ increases from 0 to $\pi$, this network experiences expansion and folding successively. When $\theta$ reaches $\frac{\pi}{2}$, the network reaches its deployed configuration (Fig. 12, c).

The networks were then simulated to identify the singularity point along with its orientation for further analysis. Singularity occurs at $\theta=150^{\circ}$ for the first network with opposite links equal in length and at $\theta=141^{\circ}$ for the second network with equilateral Bennett mechanisms. These results are novel and not attempted before by researchers. Coupler curve for both the Bennett mechanisms were plotted in MATLAB.

\section{Conclusion}

Two types of Bennett mechanisms were identified and then constructed, physically and also in MATLAB software, based on their geometrical conditions and the kinematics. The problem is to identify the type of Bennett mechanism that is most appropriate for developing deployable structures.

Type 1 and type 2 Bennett mechanisms has only four links, it's a simple mechanism and not a compound mechanism. The basic difference between two mechanism are Type 1 Bennett mechanism has opposite links of same length and type 2 Bennett mechanism has all the links of same length. Type 2 Bennett mechanism (we can also call it as Equilateral Bennett mechanism) was found to be the best when compared to type 1 for application such as deployable structure.

The problem was solved by taking both the two types of Bennett mechanism individually and constructing a physical model using PVC pipe and a nut and bolt arrangement. Also the two types were simulated in MATLAB as well. After considering individually, a network was constructed physically and using MATLAB using nine Bennett mechanisms connected together in a particular manner such that one of the Bennett mechanism placed at the centre has extended links. Then based on the minimum foldable input angle configuration, the type of Bennett mechanism which is more foldable and compact was founded. Again type 2 Bennett mechanism was the best.

The network is made of nine Bennett mechanisms combinations. When analysing the network it was found that while extending the input links from $0^{0}$ to $180^{\circ}$ it has formed a shape of a saddle. Therefore, a saddle surface was created.

Next was to obtain a perfect and expected saddle shape, the required saddle structure should be designed by choosing the appropriate link length and twist angle for Bennett mechanism. The obtained network is similar to that of a deployable structure. Deployable structures can be easily folded and are compact. Therefore their storage and trans- 
portation is also very easy. It can be deployed into their service configuration as and when required. Hence they are used in military camps and trekking camps also.

The step by step formation of the saddle surface is analysed by varying the twist angles. When the twist angle is at $20^{\circ}$, a more or less flat surface is formed. The saddle point formation is at the beginning stage. When the twist angle is increased to $30^{\circ}$, a fine saddle surface is obtained. It is found that the steepness of the saddle surface increases with increase in twist angle.

Usually saddle structures have high rigidity and are mainly used as roofs in modern buildings or as foldable tends which can be carried to any place very easily. But there is a difficulty in installing these structures, hence these Bennett networks provides a simple way for constructing modern saddle roofs. This reduces the time of installing and makes the saddle structure more perfect.

\section{References}

1. Bennett, G.T. 1914. The skew isogram, London mathematical society. 151 https://doi.org/10.1112/plms/s2-13.1.151.

2. Phillips, J. 1984. Freedom in Machinery: Volume 1, Introducing Screw Theory, Cambridge University Press, Cambridge, UK. https://doi.org/10.1017/S0263574700009206.

3. Phillips, J. 1990. Freedom in Machinery-Volume 2: Screw Theory Exemplified, Cambridge University Press, Cambridge, UK. https://doi.org/10.1017/S0263574700010523.

4. Chen, Y.; You, Z. Alternative Form of Bennett Linkage, Department Report No. OUEL 2245/01, Department of Engineering Science, University of Oxford. http://www.eng.ox.ac.uk/civil/publications/reports1/ouel_2245-01.pdf.

5. Lee, C. 1995. Kinematic analysis and dimensional synthesis of Bennett 4R mechanism, The Japan Society of Mechanical Engineers 38(1): 199-207. https://doi.org/10.1299/jsmec1993.38.199.

6. Zhi, C.; Wang, S.; Sun, Y.; Li, J. 2015. Kinematic and dynamic characteristics analysis of Bennett's linkage, Journal of Harbin Institute of Technology 22(3): 95-100. https://doi.org/10.11916/j.issn.1005-9113.2015.03.014.

7. Wei, W. 2011. Assembling and analysis of large special deployable and networks based on Bennett linkage, Master degree dissemination, Harbin institute of technology, Shen Zhen.

8. Parkin, L. A. 2010. On structural properties of sets of finite displacement screws, Adnances in robot kinematics: motion in man and machine: 493-498. https://doi.org/10.1007/978-90-481-9262-5_53.

9. Brown, M. V.; Milenkovic, P. 2011. Properties of the Bennett mechanism derived fromthe RRRS closure ellipse, Journal of Mechanisms and Robotics 3(2): 021012-1-021012-8. https://doi.org/10.1115/1.4003844.
10. Baker, J.E.; Min, H. 1986. On spatial networks of overconstrained linkages, Mechanisms and Machine Theory 21(5):427-437. https://doi.org/10.1016/0094-114X(86)90091-1.

11. Baker, J. E. 1988. The Bennett linkage and its associated quadric surfaces, Mechanisms and Machine Theory 23(2): 147-156. https://doi.org/10.1016/0094-114X(88)90092-4.

12. Yang, F.; Li, J.; Chen, Y.; You, Z. 2015. Deployable Bennett Network in Saddle Surface, The 14th IFToMM World Congress, Taipei. 428-434 https://doi.org/10.6567/IFToMM.14TH.WC.OS8.015.

13. Baker, J. E. 2013 A collapsible network of similar pairs of nested Bennett Linkages, Mechanisms and Machine Theory 59: 119-124

https://doi.org/10.1016/j.mechmachtheory.2012.09.003.

14. Bil, T. 2012. Analysis of the Bennett linkage in the geometry of the tori, Mechanisms and Machine theory 53: $122-127$. https://doi.org/10.1016/j.mechmachtheory.2012.03.003.

15. Soni, A. H. 1974. Mechanism Synthesis and Analysis, McGraw-Hill Book Co, New York.

\section{T. P. Valayil, S. Velappan, P. L. Pandian}

ANALYSING THE KINEMATIC CHARACTERISTICS OF BENNETT MECHANISMS AND ITS NETWORKS FOR USAGE IN FORMING DEPLOYABLE STRUCTURES

S u m m a r y

Two types of Bennett mechanism are encountered in most of the related literatures. This research aims at finding the best type of Bennett mechanism for making deployable structure. First type is known as equilateral Bennett mechanism having equal links. Next type of Bennett mechanism has opposite links of equal length. Using the two types of Bennett mechanism, two different networks are created. Their working range, minimum foldability and maximum foldability of the networks were identified using MATLAB. The results prove that equilateral Bennett mechanism had better foldability. Then on analyzing the shapes of the networks, it was found that the networks had a saddle shape. The obtained saddle surface provided certain results like, when the twist angles of the Bennett links were varied; saddle surfaces with more steepness are obtained. The influence of twist angle on kinematics was analyzed. Singularity analysis was also done and coupler curves were plotted.

Keywords: Bennett mechanism, networks, kinematics, deployable structures, saddle surfaces.

Received January 21, 2019

Accepted August 26, 2019 